Editorial Article

Open Access

\title{
Scrub Typhus: An Emerging and Re-emerging Metazoonosis of Public Health Significance
}

\author{
Mahendra Pal ${ }^{*}$ \\ Founder of Narayan Consultancy on Veterinary Public Health and Microbiology, 4 Aangan, Jagnath Ganesh Dairy Road, Anand-388001, India
}

\section{Article Info}

*Corresponding author:
Mahendra Pal
Founder of Narayan Consultancy
Veterinary Public Health and Microbiology
4 Aangan, Jagnath Ganesh Dairy Road
Anand-388001, India
E-mail: palmahendra2@gmail.com

Received: August 13, 2017

Accepted: August 18, 2017

Published: August 25, 2017

Citation: Pal M. Scrub Typhus: An Emerging and Re-emerging Metazoonosis of Public Health Significance. Madridge J Immunol. 2017; 1(1): 16-17.

doi: $10.18689 / \mathrm{mjim}-1000105$

\begin{abstract}
Copyright: () 2017 The Author(s). This work is licensed under a Creative Commons Attribution 4.0 International License, which permits unrestricted use, distribution, and reproduction in any medium, provided the original work is properly cited.
\end{abstract}

Published by Madridge Publishers
Keywords: Leishmaniasis; Japanese encephalitis, Scrub typhus; Clonorchiasis; paragonimiasis; trypanosomiasis; Sindbis fever.

Metazoonosis is defined as disease that is transmitted biologically by invertebrate vector such as mosquito, tick, mite, sand fly, rat flea, tsetse fly and the agent develops, multiplies or both in vectors before transmission. There are many metazonoses such as plague, dengue fever, chickungunya fever, murine typhus, yellow fever, rickettsial pox, Crimean-Congo haemorrhagic fever, ilheus fever, Mayaro fever, leishmaniasis, Japanese encephalitis, scrub typhus, clonorchiasis, paragonimiasis, trypanosomiasis, Sindbis fever, filariosis, babesiosis, Kayasanur forest disease, phlebotomus fever, West Nile fever, Rift Valley fever, borreliosis, eastern equine encephalitis, Colorado tick typhus and tularaemia, which are significant causes of high morbidity and mortality both in developed as well as developing countries of the world. Among these, Scrub typhus (chiggar borne rickettsiosis, Japanese fever, mite borne typhus fever, rural typhus, tropical typhus, Tsutsugamushi disease) is an important emerging and reemerging vector borne metazoonosis zoonosis, which is caused by Orientia (Rickettsia) tsutsugamushi, an obligate, intracellular rickettsial organism. The disease was observed for the first time in 1899 from Japan and named as Tsutsugamushi (tsutsuga means dangerous and mushi means insect or mite). Later, it was reported from Australia, India, Indonesia, Korea, Malaysia, Myanmar, Nepal, Pakistan, Russia, Sri Lanka, and Thailand. In India, scrub typhus is prevalent in many states such as Sikkim, Assam, Himachal Pradesh, Manipur, Meghalaya, Jammu Kashmir, Nagaland, Uttrakhand, Kerala, Orissa, West Bengal, Chhattisgarh, Maharashtra, Andhra Pradesh, Rajasthan, Haryana, and Delhi. Scrub typhus is a re-emerging disease in Indian subcontinent. The resurgence of disease is attributed due to several factors such as deforestation, unplanned urbanization, rapid industrialization, poor sanitation, and increased transportation. During world war second, scrub typhus was considered as a dreaded disease as it took heavy tolls of soldiers. It is estimated that 3914 cases of scrub typhus occurred in Thailand during 2000. Currently, one billion people are at risk of getting scrub typhus and about one million cases are reported every year. The case fatality used to be as high as $60 \%$ before the advent of antibiotics. The outbreaks of scrub typhus are usually observed during rainy season; however, outbreaks in cooler months are recorded from Southern India. The disease remains misdiagnosed and underreported mainly in rural settings because of poor laboratory facility. Therefore, the real magnitude of disease cannot be judged. Scrub typhus is an occupational zoonosis of forest workers, joggers, campers, gardeners, and farmers.

Human is an accidental host and acquires the infection by the bite of infected larval mite (chigger) of the genus Leptotrombidium. The adult mite does not attack on humans and feeds only on plants. It is now well recognized that chigger mite acts as vector and reservoir for Orientia tsutsugamushi and can transmit the infection to humans and other vertebrate animals including rodents. The birds may act as 
mechanical carrier of mite. The mite can survive in grassy regions, rain forests, scrub vegetation, mountain deserts, riverbanks and sandy beaches.

The incubation period of scrub typhus is 1 to 3 weeks. Most patients develop a characteristic eschar at the site of the chigger bite. The bites are mostly seen on neck, axilla, groin and genitalia. The patient exhibits signs of fever, headache, sweating, chills, vomiting, abdominal cramps, myalgia, fatigue, cough, pneumonia, deafness, conjunctivitis, lymphadenopathy, and cutaneous rash.

The presence of black eschar at the site of bite by mite larva gives a valuable clue to the physician to make a tentative diagnosis. The absence of eschar presents a challenge to the clinician in diagnosis. However, a number of laboratory techniques such as microbiological, immunological and molecular are employed to confirm the diagnosis of scrub typhus. Presently, Weil Felix test is not used for diagnosing the disease. The organism can be cultivated on L929 cells and can be stained with Giemsa technique. The isolation of pathogen from clinical specimens is tedious and time consuming and requires BSL3+ facility in the laboratory. Hence, indirect immunofluorescence assay is considered the mainstay for serodiagnosis of disease. Among the molecular tools, real time PCR and nested PCR are rapid and sensitive tests but are not easily available in most of public health laboratories. Hence, attempts should be made to develop simple and low cost test, which can be easily applied at the primary health centers located in rural and suburban areas. Scrub typhus should be differentiated from malaria, typhoid fever, dengue fever, murine typhus and leptospirosis.
A number of drugs such as azithromycin, chloramphenicol, doxycycline and minocycline are available for the management of disease. Doxycycline is most widely used as therapeutic agent to treat cases of scrub typhus. In certain areas where doxycycline alone does not show good clinical response, it is advised to go for combined therapy with doxyxycline and rifampicin. However, doxycycline is contradicted in children. Therefore, chloramphenicol can be safely tried in children and pregnant women. In untreated patients, death may occur due to cardiac failure, meningitis and pneumonia.

The eradication of scrub typhus seems unfeasible in the absence of potent vaccine that can induce lifelong immunity. Therefore, early diagnosis and prompt specific therapy, wearing of protective clothing, use of repellent cream on exposed parts of body, clearing of vegetation, application of acaricides such as lindane in high risk areas, rodent control and public health education will certainly mitigate the problem of scrub typhus. It is pertinent to mention that persons visiting hyperendemic areas should take doxycycline $200 \mathrm{mg}$ capsule daily for one week as a prophylaxis to protect from infection.

Currently, no commercially produced vaccine is available to immunize the susceptible population in endemic regions of the world. Therefore, attempts should be directed to develop safe, potent and low cost vaccine, which can be easily afforded by poor resource nations for immunizing the susceptible population against this metazoonosis. It is emphasized that persons in endemic areas with fever and history of bite from mite should be thoroughly investigated for scrub typhus. It is adised that diagnostic kit and drugs should be provided at cheaper prices in endemic regions of poor countries to reduce the chances of life threatening complications due to scrub typhus. 